0031-3203(94)E0018-G

\title{
SUBPIXEL PATTERN RECOGNITION BY IMAGE HISTOGRAMS
}

\author{
TAMÁs SZIRÁnYi \\ Analogical and Neural Computing Systems Research Laboratory, Computer and Automation Institute, \\ Hungarian Academy of Sciences (MTA SZTAKI), P.O. Box 63, H-1518 Budapest, Hungary
}

(Received 6 January 1993; in revised form 1 February 1994; received for publication 24 February 1994)

\begin{abstract}
Recognition of small patterns covering only a few pixels in an image cannot be done by conventional recognition methods. A theoretically new pattern recognition method has been developed for undersampled objects which are (much) smaller than the window-size of a picture element (pixel), i.e. these objects are of subpixel size. The proposed statistical technique compares the gray-level histogram of the patterns of a set of scanned objects to be examined with the (calculated) gray-level densities of different (in shape or size) possible objects, and the recognition is based on this comparison. This method does not need high-precision movement of scanning sensors or any additional hardware. Moreover, the examined patterns should be randomly distributed on the screen, or a random movement of camera is (or target or both are) needed. Effects of noise are analysed, and filtering processes are suggested in the histogram domain. Several examples of different object shapes (triangle, rectangle, square, circle, curving lines, etc.) are presented through simulations and experiments. A number of possible application areas are suggested, including astronomy, line-drawing analysis and industrial laser measurements.
\end{abstract}



\section{INTRODUCTION}

Subpixel measurement technology is a new field in digital image processing. Several methods have been developed to detect some specific features ${ }^{(1-4)}$ of image segments of low resolution. In these measurements the accuracy is higher than the scanning resolution of the camera.

One of the main areas of subpixel technique is the position analysis of line drawings. Koplowitz and Sundar Raj ${ }^{(5)}$ propose a method which reconstructs the original curve from chain coded data. When a straight line segment has been digitized into a chain code sequence with $N$ chain points and the distance between the pixels is $D$, then the subpixel resolution is about $D / \sqrt{ } N$. Lyvers et al. ${ }^{(3)}$ determine the position of an edge with an accuracy of about $1 / 20$ pixel by a moment-based edge operator to measure imaged metal machine parts. The effect of noise is also considered. It is found that the operator has a relatively small bias in the presence of noise. Cox et al. ${ }^{(4)}$ use matching methods to predict and estimate locations of edges with an accuracy of $3 \%$ interpixel spacing. Alexander and $\mathrm{Ng}^{(6)}$ show when the Nyquist criterion is satisfied for a scanned object, the location of the object can be estimated by a subpixel accuracy centroid method. Reichenbach et al. ${ }^{(1)}$ characterize digital image devices using edge detections of subpixel accuracy. Huertas and Medioni ${ }^{(7)}$ have developed a subpixel operator using the facet model combined with Laplacian-
Gaussian masks. Warmerdam and Algazi $^{(2)}$ give a method to describe the edge transition by Gaussian Derivatives at the resolution matching the transition widths. Aghajan et al ${ }^{(8)}$ use neural networks to estimate the multipixel line-width of straight lines and to measure their position by subpixel accuracy in microlithography. Szirányi et al..$^{(9,10)}$ show an inverse statistical method to estimate the diameter of a laser beam by a moving sensor-array by subpixel accuracy. Szirányi ${ }^{(11)}$ suggests the same technique for parameter estimation of nearly noiseless light-spots in two dimensions.

Conventional pattern recognition and other image analysis methods deal with objects which can be described by several pixels and gray-levels. In the spatial frequency domain the main criterion to appropriately describe an object is the Nyquist criterion: the spatial frequency which corresponds to the scanning resolution should be at least twice the spatial bandwidth of the image (reference 12, p. 97). Sometimes, the spectrum folding of the aliasing effect can be exploited as an information source in the lower spectra, ${ }^{(13)}$ but in general the aliasing effect degrades the image. In the case of low resolution pictures ${ }^{(14,15)}$ the recognition works on a limited number of classes only. When the object to be recognized is so small that it covers few pixels only, the conventional statistical or syntactical recognition methods will no longer work correctly.

However, the well-known and precise characteristics of recent image capture devices give new possibilities 
for low-resolution picture processing. For about 10 years new types of scanning arrays (e.g. CCD $=$ Charge Coupled Device, ${ }^{(16-18)}$ photodiode array ${ }^{(18)}$ have been available. They have some properties which are important in recent applications: the elementary scanning windows are nearly independent of their neighbors in the photoarray, the geometry and sensitivity distribution of the elementary scanning windows are the same for all windows and these properties are given by the data sheets..$^{(16-18)}$ The latest types of available image sensors have impressing characteristics: $110 \mathrm{~dB}$ dynamic range and 52-electron noise. ${ }^{(19)}$

Typically, the resolution of image acquisition devices (cameras, scanners) is limited by the scanning photomatrix and not by the optical systems; consequently, these devices are designed for undersampling. ${ }^{(1)}$

These features of the new devices give an opportunity to handle the picture information by statistical and analytical methods to get super-resolution. Moving the object with accurately measured small steps in the scanning field of the camera, the object can be scanned through a convolution. ${ }^{(1)}$ Similarly, the position or angle of a known large (multipixel) shape (e.g. an edge) can be measured by analytical subpixel algorithms. ${ }^{(1-4)}$ However, nothing has been published on the recognition of small subpixel-size objects.

Now, a new method will be presented for the recognition of subpixel objects. In some applications we have very small (relative to the pixel window) patterns on the screen originated from objects of the same shape and size, but we cannot recognize them with conventional pattern recognition methods. It is also possible that we know the shape of the scanned objects, but the diameter or some other parameter of these scanned similar objects should be determined.

One of the main areas of subpixel technique is the line drawing analysis. In this case not only the positions of line-segments, ${ }^{(5)}$ but also the line-width and type (continuous or dotted) can be determined by this subpixel recognition/measurement technique from undersampled images.

As an example, one can think of the case when the distance between the stars of a double star is to be estimated in an undersampled astronomical measurement, where the spots of the optically projected stars and their distance are smaller than an elementary scanning-window of the scanning-matrix of the camera, and the optical magnification cannot be increased (e.g. on a space telescope). Here, the histogram statistics are provided by the results of image sequences about the stars at random positions (the telescope is not stabilized firmly in the space).

Another example is provided by simultaneous widerange tracking and beam-spot measurement ${ }^{(11)}$ or determination of a laser beam. Following the track of a light beam (laser beam) is an important task in many laser-optical measurements (cutting and welding with laser, optical-memory devices). In these cases the measured track runs in an image area that is much larger than the width of the beam-spot.
A non-visual image analysis example is robot tactile sensing. ${ }^{(15)}$ The relatively small objects contacted randomly with the sensor array or the quality of surface could be determined by this subpixel method.

The exact theoretical grounds of this new method will be shown in this paper. Effects of noise, application possibilities and the practical limits will be analysed by simulations and experiments. It will be shown that not only the subpixel-accurate position of a multipixel shape, but the exact recognition of such subpixel-size objects can be done by statistical methods, without high-precision moving or additional hardware. The recognition needs only a gray-level statistics (histogram) of the objects to be determined. These objects frequently appear randomly and separately on the screen, or there is a histogram made of consecutive expositions about the same randomly moving object.

Histograms are often used in picture processing. They are the gray-level statistics of the picture. In the normalized case the histogram is the estimation of the probability density function of the gray-levels of the pixels in the image. Making histograms ${ }^{(20)}$ is one of the basic steps of any picture processing system (both in hardware and software), ${ }^{(12,21)}$ so our method is easy for any applications. However, this new histogrambased technique cannot be derived from the conventional statistical pattern recognition methods which also use image histograms.

In our case the shape and the size of the measured objects are the same in the whole detected area. The light-sensitivity of elementary sensor-windows is uniform (or it can be made uniform by memorized pixelbias values), and the reflection factor of the targets (or the transmission factor) is homogeneous. The objects projected onto the sensory array may be much smaller than the width of an elementary scanning-window. Then, we can use a new statistical measurement method to recognize the subpixel-size objects from their projected patterns in the image. This method is based on a histogram-evaluating method which makes the graylevel statistics (histogram) of the scanning-windows (pixels) (partly or fully) covered by the scanned object. Prior to the measurement, the possible object classes must be considered in the calculations of gray-level densities based on the known sensitivity distribution of the scanning windows and the light-intensity distribution of the detected objects. During the recognition, the histogram of the measured patterns and the considered densities of the different object classes are compared, and the class of the most similar density is given as the result.

In the following sections the original theory of subpixel pattern recognition (model, calculation of densities, comparison of measured histogram and considered densities, effects and removal of noise in the histogram domain) will be presented, then experimental results are shown. Elementary shapes (as circles, double circles, rectangles, etc.) will be presented for subpixel recognition. The theoretical limits of the method will be shown in the spatial and the frequency domain as well. 


\section{THE THEORY}

Consider an input picture with the separated patterns of the same object class or a sequence of pictures with the patterns of the same object class. The scanned objects are small compared to the pixel width. It means that there is only one or a few gray-level values in a pattern when an object is scanned. Let us suppose that a scanning-window is not covered by more than one subpixel object at the same time.

In the case when there are enough samples of such subpixel pattern and the image sources are statistically stationary and ergodic, it will be shown that the class of the scanned objects can be determined by evaluating the histograms of the measured patterns.

\subsection{Calculation of densities}

In the first step, the theoretical distribution of possible gray-levels is calculated by computer simulation for the objects to be detected. Let $S(x, y)$ denote the photosensitivity distribution of an elementary sensory window (see Fig. 1). ${ }^{(13)} L(x-\Delta x, y-\Delta y \mid \omega)$ is the lightintensity distribution of the measured $\omega \in \Omega$ object at $(\Delta x, \Delta y)$ characteristic distance from the window's reference point, $R($.$) is the response function of an ele-$ mentary scanning pixel window, and $\Omega$ is the set of the different object classes. Then, the photo-exposition (the gray-level of pixel) for an $(i, j)$ indexed elementary sensor-window of the sensor-matrix is a function of the window-convolution and the response function:

$$
\begin{aligned}
& G_{i, j}(\Delta x, \Delta y \mid \omega) \\
& \quad=R\left(\int_{x} \int_{y} S(x, y) L(x-\Delta x, y-\Delta y \mid \omega) \mathrm{d} y \mathrm{~d} x\right) .
\end{aligned}
$$

Here the integral is interpreted in the whole $(x, y)$ area where the $S(x, y)$ sensitivity function of one scanning-window is considered nonzero. When the used sensor accumulates the light-power between exposures (as the TDI-CCD ${ }^{(22)}$ does), a time-domain integration must also be considered in convolution (1) ${ }^{(9)}$ Generally, the response function $R($.$) is a power function (refer-$



Fig. 1. A sensor window of scanner (camera) with its close neighbors and the measured object spot projected onto the sensor-matrix ence 21, p. 273) of the illumination of the sensory window. However, the new types of image capture devices (e.g. CCD arrays ${ }^{(16-19)}$ have linear characteristics in a wide, 2000-100,000 dynamic range. Since in most cases $R($.$) may be considered a linear function of$ illumination, in the following part the window responsefunction $R($.$) in equation (1) will be considered linear$ $(R()=1.0$.$) . For stationary and ergodic images the$ index $(i, j)$ may be omitted.

If both $\Delta x$ and $\Delta y$ have uniform probability distribution $(\Delta x, \Delta y$ and the $(i, j)$ positions of the exposition are independent), the $h(G \mid \omega)$ probability density of $G(\omega)$ can be calculated in the $(G, \Delta x, \Delta y)$ space. The $G(\Delta x, \Delta y \mid \omega)$ function is cut by the $G(\Delta x, \Delta y \mid \omega)=G_{0}$ and the $G(\Delta x, \Delta y \mid \omega)=G_{0}+\Delta G$ planes. The projections of this section of the $G(\Delta x, \Delta y \mid \omega)$ surface to the $(\Delta x, \Delta y)$ plane is proportional to the probability of $G \in\left[G_{0}, G_{0}+\Delta G\right)$. When $\Delta G \Rightarrow 0$, we obtain the $h(G \mid \omega)$ density. When $\Delta G$ means discrete finite intervals, we obtain the probability distribution (PD).

If $|\mathrm{d} G(\Delta \mathbf{r} \mid \omega) / \mathrm{d} \Delta \mathbf{r}|^{-1}$ exists and is measurable, where $\Delta \mathbf{r}=(\Delta x, \Delta y), \Gamma=\left\{\Delta r \mid G(\Delta r \mid \omega)=G_{0}\right\}$, and the following integral exists in the Lebesque-sense, it can be shown that the density function is

$$
h\left(G_{0} \mid \omega\right)=\int_{\mathbf{\Gamma}} p_{\Delta \mathbf{r}}(\Delta \mathbf{r})\left|\frac{\mathrm{d} G(\Delta \mathbf{r} \mid \omega)}{\mathrm{d} \Delta \mathbf{r}}\right|^{-1} \mathrm{~d} \Gamma .
$$

The density $p_{\Delta r}$ of $\Delta \mathbf{r}$ is usually considered uniform.

As a demonstration, in Fig. 2 the coverage $G(\approx$ photoexposition) of a pair of small circles at a distance of $40 \%$ of interpixel spacing is shown as a function of the $(\Delta x, \Delta y)$ interpixel position.

Practically, in the computer calculation, in the case of every calculated $\omega$ class, the $(\Delta x, \Delta y)$ area is divided into small equal sections, and direction $G$ is also divided into intervals. For every interval of $(\Delta x, \Delta y)$ area the quantized $G$ is calculated from equation (1) and the counter of the interval according to the calculated $G$ value is incremented by one. When the partition of $(\Delta x, \Delta y)$ subspace is not equidistant, the counter of summation should be incremented by the measure of the examined $(\Delta x, \Delta y)$ interval-section.

Since there are positions where an object covers two or more $(i, j)$ pixel-windows at the same time (when it is on the border among windows), it gives some statistical dependencies among the different exposition values of the same position. Because of these dependencies, some neighborhood of the windows should be taken into consideration in the calculation, too. In this case, at a given position $(\Delta x, \Delta y)$ of the projected object relative to the reference sampling-window, the neighbors of this window are also considered in statistics with their exposure values. It means that in the case of the nearest neighborhood, 9 pixel values should be calculated in a $(\Delta x, \Delta y)$ position. If it is necessary, the interaction effect of the neighboring pixels can also be taken into consideration by this method.

Not only $\Delta x$ and $\Delta y$, but also the rotation angle $\alpha$ of the object can be calculated in this way, $\Delta \mathbf{r}=$ $(\Delta x, \Delta y, \alpha)$. 


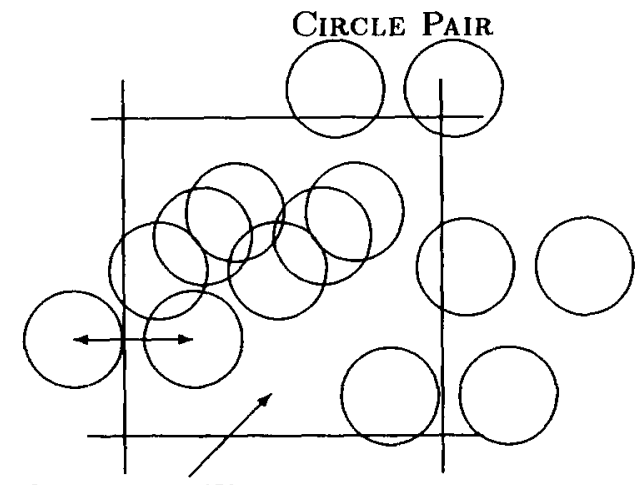

SCANNING Window
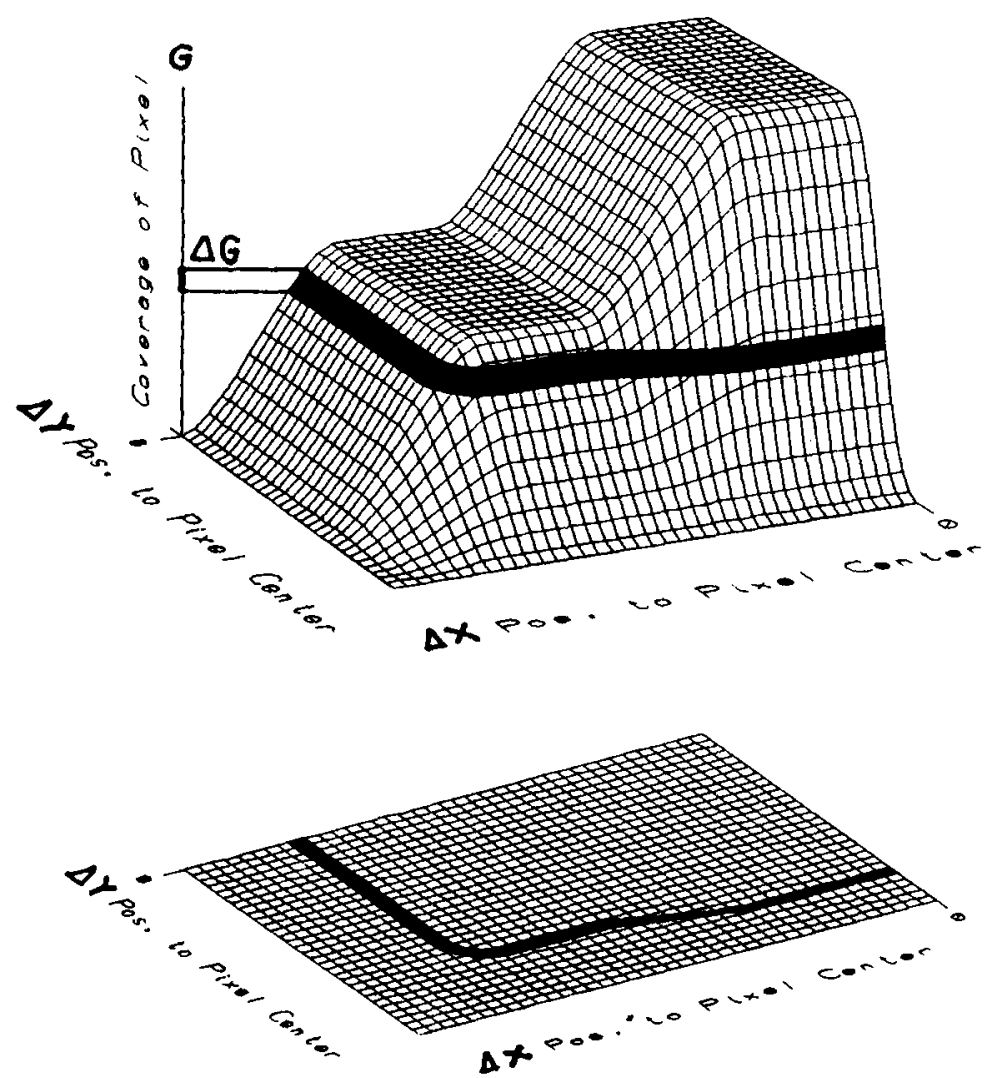

Fig. 2. Probability calculation of $P\left(G \in\left[G_{0}, G_{0}+\Delta G\right]\right)$ from the $G(\Delta x, \Delta y \mid \omega)$ function in the $(G, \Delta x, \Delta y)$ space. The probability is proportional to the projected area of the $G \in\left[G_{0}, G_{0}+\Delta G\right]$ interval intersection of the $G(\Delta x, \Delta y \mid \omega)$ function to the $(\Delta x, \Delta y)$ plane.

The local inhomogenity of transition width at the border of the scanning windows influences the histogram statistics very slightly, as it was simulated for line CCD sensors. ${ }^{(9)}$ When the rotation of object is not considered, the small difference between the estimated and measured angle positions of the object may cause an error in the measurement. ${ }^{(22)}$

When more subpixel-scale objects may be projected into the same pixel-window and their position relative to each other is known or statistically well-defined, this condition can be involved in the calculations. (See the experiments of single and double spots, Figs 3 and 4.)
A fixed group of subpixel objects should be handled as one object. When patterns are periodically spaced in the image, it results in effects as in Figs 5 and 6 for periodical dots and lines. Such types of repetition should be considered in the calculation of densities.

\subsection{Classification: comparing the histogram and densities}

In the measurement we take the histogram of the gray-levels $G$ of the measured similar objects, and this histogram $h(G)$ is compared, as an estimation, with the 




Fig. 3. Histograms of circular spots of different relative areas.



(a)

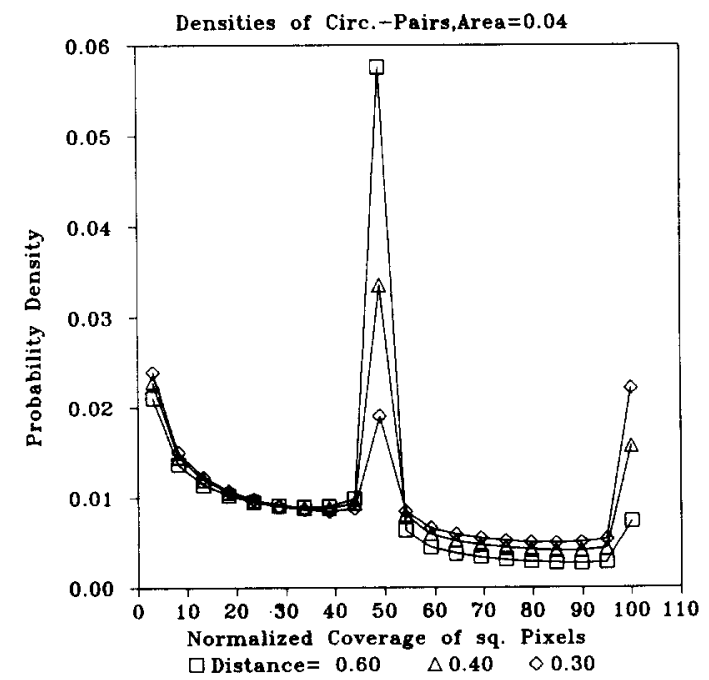

(b)

Fig. 4. (a) Histograms of circular spot-pairs of 0.04 relative area and different relative center-distances. (b) Densities of circle-pairs.

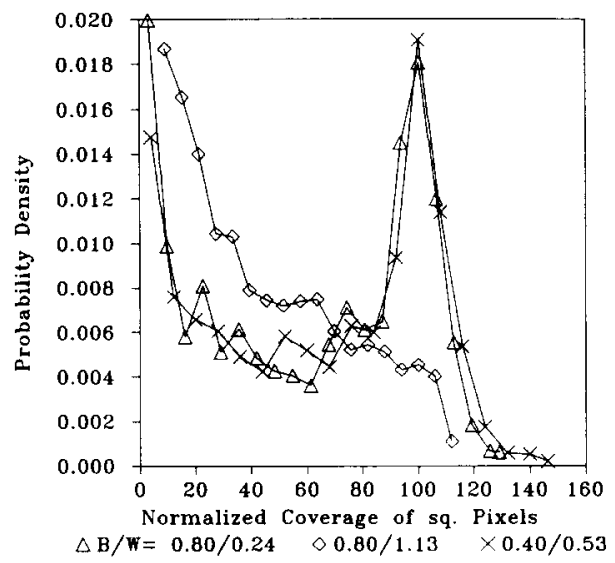

Fig. 5. Histograms of curving lines of different black/white ratios. The parameter is the relative length of black/white sections. Line-width is 0.40 .

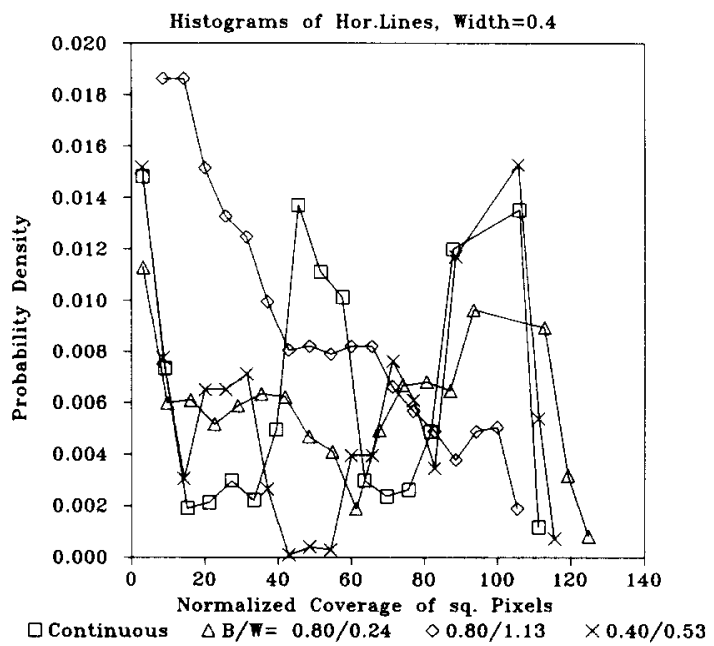

Fig. 6. Histogram of horizontal lines of different black/white ratios. The parameter is the relative length of black/white sections. Line-width is 0.40 .

calculated $h(G \mid \omega)$ densities for every $\omega \in \Omega$ object class. Estimating densities is a well-known area of statistics. ${ }^{(23-26)}$

The histogram and the densities are compared by some discriminant functions. The classification is taken on the results of these comparisons, as the maximum of Correlation and minimum of L1-distance ${ }^{(25,27)}$ (absolute integral of difference of densities) and information divergences. ${ }^{(23)}$

This classification method, when the measured histogram and densities of different classes are compared to estimate parameters through nonparametric estimation, is relatively new. Partly as a consequence of the present work, some latest results deal with the robustness and consistency of this method. ${ }^{(27)}$

The correlation is considered between the measured $h(G)$ and the calculated $h(G \mid \omega)$ functions for $G \in K$ :

$$
\text { Corr }=\frac{\int h(G) h(G \mid \omega) \mathrm{d} G-1}{\sigma_{h(G)} \sigma_{h(G \mid \omega)}},
$$


where the standard deviations for $h(G)$ in the normalized case are in the form:

$$
\sigma_{h(G)}=\sqrt{\left(\int(h(G)-1)^{2} \mathrm{~d} G\right) .}
$$

It is similar for $\sigma_{h(G \mid \omega)}$ of $h(G \mid \omega)$.

Here $K$ is the common interpretation interval of $h(G)$ and $h(G \mid \omega)$ normalized functions, which are normalized again to get the integral be equal to 1.0 for them over the $K$ interval domain. The definition of the Kullback-Leibler divergence for densities $f$ and $g^{(28)}$ for $G \in K$

$$
D(f \| g)=\int f(G) \log \left(\frac{f(G)}{g(G)}\right) \mathrm{d} G .
$$

When $D(h(G) \| h(G \mid \omega))$ divergence is minimized over $\omega$, the result is similar to that of the maximum-likelihood (ML) method. ${ }^{(29)}$ When $D(h(G \mid \omega) \| h(G))$ is minimized over $\omega$, we obtain the Kullback minimal discriminant information (MDI). ${ }^{(29)}$

When the minimal information divergence is relatively small for the winner class, the estimation is sufficient. This sufficiency can be estimated from the number of measurements, the hypothetical and the measured probabilities and the $\chi^{2}$ distribution, ${ }^{(29)}$ since the

$$
N \sum_{k=1}^{r} \frac{\left(H\left(G_{k}\right)-H\left(G_{k} \mid \omega\right)\right)^{2}}{H\left(G_{k} \mid \omega\right)}
$$

normalized quadratic estimation error converges to the $(r-1)$ th order $\chi^{2}$ distribution, when the number of independent measurements $N \Rightarrow \infty .^{(29,30)}$ Here the number of $G_{k}$ intervals is $r$, and the $H\left(G_{k}\right)$ and $H\left(G_{k} \mid \omega\right)$ probabilities are interpreted as the integrals of $h(G)$ and $h(G \mid \omega)$ densities over the $k$ th $(1 \leq k \leq r)$ interval of $G$.

When more than one class give sufficient results with $\chi^{2}$ test, it means that these classes must be considered one identical class. In order to increase the number of separable classes, the number of $G_{k}$ intervals (the measurement accuracy) has to be increased. There can be cases, however, when possible patterns of two different object classes give (nearly) the same statistical densities that can be tested through density simulations.

Since the result of $\chi^{2}$ test and the maximum-likelihood ratio test are equivalent, ${ }^{(29)}$ and for the L1 distance and information divergence of two probabilities there is a bouding criterion (reference 31, p. 58), the above discriminant functions and $\chi^{2}$ test are in close connection. However, the correlation method is not invariant to scaling, while the others are (the L1distance and the informational distances are $f$ divergences). (23,27) $^{(2.3 .}$

\subsection{Effects of image noise and background in the histogram domain}

Since subpixel signals mean a special type of "noise", taking the effects of other noise sources into consideration is a crucial point of this method. Reduction of noise influence can be achieved through the appropriate noise models.
The first experiments ${ }^{(32)}$ were made with images from high-quality scanners, where the images were uniformly colored black drawings on clean white paper sheets. Even in this ideal case some moderate effects of noise have been found. However, one of the main application possibilities of this technique is in the analysis of undersampled line-drawings where, fortunately, it is not necessary to deal with high noise.

In a very general case the effects of noise and uneven background should be taken into consideration. Filtering the picture, transformations can be applied only which do not influence the useful information content of histograms. Generally, the image-filtering methods (averaging, edge-enhancement, noise-removing) change the information content of both the image and the histograms, ${ }^{(20)}$ so they cannot be used here.

In many cases pixels of subpixel patterns can be separated by some edge detection ${ }^{(12)}$ or target-following algorithm. The enhanced pixels can be separated, and the original (non-filtered) pixel-values $(G)$ of the separated pixels should be taken into the histogram. Separating the edge pixels and their close neighbors, the remaining pixels can be considered the background. The statistics of background and the noise effects can be estimated from the separated background. On the basis of background parameters, a background-equalization can be processed on the whole image (containing also the subpixel points). In this way, we can get more correct histogram statistics of the supposed subpixel patterns. This histogram may contain some effects of noise and the effects of background. In this section we deal with noise removal of histograms and estimation of noisy densities in the histogram domain, too.

Two main types of noise and background are considered: additive noise and multiplicative noise. Now, the uneven background is considered the main source of noise.

In the first case a noisy (or uneven) background is covered by the object examined (e.g. in astronomical examples). The noise and the background are considered independent of the effects of the subpixel patterns. Equation (1) will be modified to be

$$
\begin{aligned}
& G_{i, j}(\Delta x, \Delta y \mid \omega) \\
& \quad=\iint_{\mathscr{A} \text { in }(i, j)} S(x, y) L(x-\Delta x, y-\Delta y \mid \omega) \mathrm{d} y \mathrm{~d} x \\
& \quad+\iint_{\mathscr{A} \text { out }(i, j)} S(x, y) B(x, y) \mathrm{d} y \mathrm{~d} x
\end{aligned}
$$

where $(x, y) \in \mathscr{A}_{\text {in }}(i, j)$ is the area covered by the object in $(i, j)$ scanning-window; $(x, y) \in \mathscr{A}_{\text {out }}(i, j)$ is the area that is not covered in the $(i, j)$ scanning-window, and the whole active domain of a scanning-window is $\mathscr{A}_{\mathrm{sw}}$ $\left(\mathscr{A}_{\text {sw }}=\mathscr{A}_{\text {in }}(i, j)+\mathscr{A}_{\text {out }}(i, j)\right)$. The light-intensity distribution of background is denoted by $B($.$) . When the$ background is uniform and the image is noiseless, the presence of background is arising as a Dirac-delta at the background intensity in the measured $h(G)$ distribution of the image. Neglecting this background peak, the first integral in equation (5) is considered the $Q$ "ideal" subpixel exposure signal at $(i, j)$ indexed sensor 
in sensor-matrix:

$$
Q_{i, j}=\iint_{\operatorname{An}^{(i, j)}} S(x, y) L(x-\Delta x, y-\Delta y \mid \omega) \mathrm{d} y \mathrm{~d} x .
$$

Restricting the investigation to the case when uniform object intensity and uniform window sensitivity are considered for a sensor at $(i, j)$ position:

$$
\begin{aligned}
& L(x, y)= \begin{cases}L, & \text { when }(x, y) \in \mathscr{A}_{\mathrm{in}}(i, j) \\
0, & \text { when }(x, y) \in \mathscr{A}_{\mathrm{out}}(i, j)\end{cases} \\
& S(x, y)= \begin{cases}S, & \text { when }(x, y) \in \mathscr{A}_{\mathrm{sw}} \\
0, & \text { when }(x, y) \notin \mathscr{A}_{\mathrm{sw}}\end{cases}
\end{aligned}
$$

and approximating $B(x, y)=B_{i, j}$ by uniform background intensity within an $(i, j)$ indexed single sensorwindow, equation (5) can be rewritten as

$$
G_{i, j}=B_{i, j} S \mathscr{A}_{\mathrm{sw}}+Q_{i, j}\left(1-\frac{B_{i, j}}{L}\right) .
$$

It is seen that the noisy exposure $G_{i, j}$ can be originated from noiseless exposure $Q_{i, j}$ through additive and multiplicative noise.

The other, very general case (e.g. black drawing on white paper) is considered when there are objects with $\Psi_{\text {obj }}$ light-reflectivity on a background of $\Psi_{\text {Backg }}$ lightreflectivity. The noisy light intensity of incident illumination is approximated to be uniform in a scanningwindow, $I(x, y) \approx I_{i, j}$. The resulted exposure value is:

$$
\begin{aligned}
G_{i, j}= & I_{i, j}\left(\iint_{\mathscr{A}(\mathrm{n}(i, j)} \Psi_{\text {Obj }} S(x, y) \mathrm{d} y \mathrm{~d} x\right. \\
& \left.+\iint_{\mathscr{O} \text { out }(i, j)} S(x, y) \Psi_{\text {Backg }} \mathrm{d} y \mathrm{~d} x\right)
\end{aligned}
$$

Considering $S(x, y)$ as earlier, we get

$$
G_{i, j}=I_{i, j} \Psi_{\text {Backg }} S \mathscr{A}_{\mathrm{sw}}+I_{i, j} \Psi_{\text {Backg }} S \mathscr{A}_{\mathrm{sw}} Q_{i, j}
$$

where the ideal noiseless exposure:

$$
Q_{i, j}=\left(\frac{\Psi_{\mathrm{Obj}}}{\Psi_{\text {Backg }}}-1\right) \frac{\mathscr{A}_{\mathrm{in}}(i, j)}{\mathscr{A}_{\mathrm{sw}}} .
$$

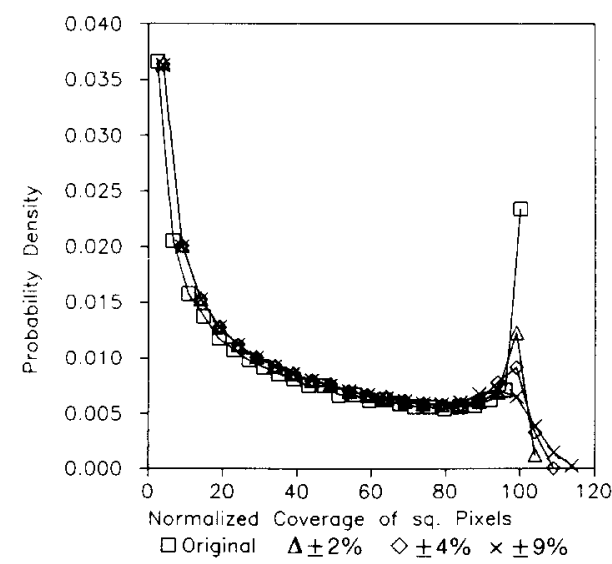

Fig. 7. Densities convolved by multiplicative noise of different standard-deviations when only the noisy $\Psi_{\text {obj }}$ is considered.
Thus, $G_{i, j}$ can be derived from $Q_{i, j}$ through the same additive and multiplicative noise variable,

$$
N_{i, j}=I_{i, j} \Psi_{\text {Backg }} S \mathscr{A}_{\mathrm{sw}} .
$$

When the lighting is uniform, $I_{i, j}=I_{0}$, and the quality of target-reflection $\Psi_{\mathrm{Obj}}$ is changing, we get a similar equation with only a multiplicative noise part (see Fig. 7).

As it can be seen from equation (7), the "ideal" exposure is a linear function of contrast $\left(\Psi_{\mathrm{Obj}} / \Psi_{\text {Backg }}\right)$. This parameter may be measured, but it is not necessary when the histograms are transformed into some normalized form (see the experiments in Section 3). This transformed function must not be dependent on the absolute lighting and contrast parameters. The normalization of a measured noisy histogram is, naturally, an approximation only, and this normalization process needs some knowledge about the general characteristics of the histogram examined.

Since, in both equation (6) and (7) the additive and multiplicative noises contain the same variable, they can be implemented in single-variable model. For the case of equation (7), we get the simple general equation for the pixel gray-levels:

$$
G_{i, j}=Q_{i, j} N_{i, j}+N_{i, j}
$$

Histograms are the estimated probability density functions of image gray-levels, noise gray-levels or their functions. Applying the general relationships for densities of continuous and derivable variables $u, v, w$ :

$$
h_{v}(v)=\int_{-\infty}^{+\infty} h_{w, u}(w, u(v))\left|\frac{\mathrm{d} u}{\mathrm{~d} v}\right| \mathrm{d} w
$$

(from reference 30 , chapter 18.5 ), and considering the independence of $Q_{i, j}$ and $N_{i, j}$, we get that for the corresponding probability densities of $G_{i, j}, Q_{i, j}$ and $N_{i, j}$ positive variables:

$$
\begin{aligned}
h_{Q}(Q) & =\int_{0}^{+\infty} h_{N, G}(N, Q N+N)|N| \mathrm{d} N \\
& \approx \int_{0}^{+\infty} h_{N}(N) h_{G}(Q N+N)|N| \mathrm{d} N \\
h_{G}(G) & =\int_{+\infty}^{+\infty} h_{N, Q}\left(N, \frac{G-N}{N}\right)\left|\frac{1}{N}\right| \mathrm{d} N \\
& =\int_{+\infty}^{+\infty} h_{N}(N) h_{Q}\left(\frac{G-N}{N}\right)\left|\frac{1}{N}\right| \mathrm{d} N .
\end{aligned}
$$

It may be approximated for the histograms interpreted as the statistics of the variables over the exposures of the sensor-elements in $(i, j)$ positions.

While the bivariate density in the first integral of equation (10) is separable, it is not true for the histogram of equation (9), because the noisy signal $G$ is dependent on noise $N$. Moreover, there is a proof of consistency for the additive-noise only, when histogram-deconvolution in density estimation is examined in reference (33). Nevertheless, a good model of noise effects help in noise removal or filtering. 
Practically, it has been found in most cases that at the high noise content of $G$, the approximate formula in equation (9) is no longer valid. So, only the use of formula (10) is acceptable, and the densities can be used in the comparison after the corruption of noise $(N)$ through equation (10). The $h_{N}(N)$ noise histogram could be estimated from the noisy $h_{G}(G)$ histogram or the background histogram of the image. The disadvantage of this method is the extra computation required for calculating noisy densities for every $\omega$ density class with the estimated noise-density.

In a very general noise model, when the ideal subpixel signal $Q_{i, j}$, multiplicative $N_{i, j}^{*}$ noise and additive $N_{i, j}^{\mathscr{d}}$ noise are independent, the general image exposition signal can be interpreted in the following way:

$$
G_{i, j}=Q_{i, j} N_{i, j}^{\mu /}+N_{i, j}^{\mathscr{d}}
$$

The density of $G$ can be obtained from the independent densities of $Q, N^{\mathscr{A}}$ and $N^{\star /}$ through some convolutiontype integrals [using equation (8) in consecutive steps for different noises] when $G, u, v>0$ :

$$
h_{G}(G)=\int_{+0}^{\infty} \int_{+0}^{\infty} h_{Q}(u) h_{N}^{\mu}\left(\frac{G-v}{u}\right)\left|\frac{1}{u}\right| h_{N}^{\mathscr{\alpha}}(v) \mathrm{d} u \mathrm{~d} v .
$$

Therefore, in a very general case, it can be said that noisy density $h_{G}(G)$ can be estimated by numerical methods from the estimated noise densities and the calculated ideal density $h_{Q}(Q)$.

\section{EXPERIMENTS}

In the following part square pixel windows of unit area are considered without gap between the neighboring pixels.

In the simulations, a pixel is considered with its 8 neighbors. It means that for a $(\Delta x, \Delta y)$ position of an object relative to the pixel center, overlaying into the close neighbors is also taken into statistics. In the simulation the rotation angle of the object is considered uniform for every $(\Delta x, \Delta y)$ position, so the gray-level is calculated in the space of three variables.

In the experiments the measurements are done by using black-typed or laser-printed patterns on white paper, scanned by image-scanner of $200 \mathrm{dpi}$ resolution. The lower resolutions are given by the numerical smoothing of the original $200 \mathrm{dpi}$ picture. In the experiments this ensures the "ideal" square pixels without neighborgap at the border. About 1000-3000 patterns have been detected for one histogram.

In the evaluation the measured histograms are parameterized relative to the hypothetical $1.0 * 1.0$ pixel dimensions. The measured histograms and the computed densities are normalized to have the same (1.0) integral, and the abscissa is also normalized in a way to bring the different histograms and densities into a comparable form.

In the figures, usually the left end is white reference. Exposition value $G$ is analogous to the coverage rate of the sensor-window.
In the comparison of hypothetical densities and a measured histogram, in most cases, for the winner $\omega$ class hypothesis, the $\chi^{2}$ test proved to be acceptable at $80-99 \%$ confidential interval assumption. The $\chi^{2}$ test shows whether the measured histogram can be accepted or more measurements (and maybe a new division of $G_{k}$ intervals) are needed. The $G_{k}$ interval division of the density is similar to the interval division of the measured histogram in the comparison.

Histogram filtering is not used in this demonstration. However, the measured and hypothetical functions are fitted considering the noise-effect. Figure 7 shows hypothetical densities convolved by estimated multiplicative noise when only the noisy $\Psi_{O b j}$ is considered. The parameter is the standard deviation of the noise of the target-reflection. This figure and further simulations demonstrate that not the right-hand-side peak but the beginning of this peak could be joined in fitting the functions together. In our comparisons the derivative before the right-hand-side peak is used in joining the functions. The noise of target-reflection is estimated as it is about $3 \%$.

In this section the basic shapes: triangles, rectangles, squares, hexagons, circles, double squares and double circles are demonstrated in simulation and experiment. These examples show that this subpixel method is capable of both pattern recognition and pattern parameter estimation.

In Fig. 8 the densities of different subpixel shapes of the same area are shown. It is seen that the densities are different, but the hexagon and circle are so close to each other that there is no real chance to identify them in our noisy experiments at the given accuracy.

Both in the calculations and the measurements, the number of gray-level intervals is 70-200. In the figures the histograms and densities are shown in maximum 30 intervals of $G$. It is easily seen that only 4-5-bit

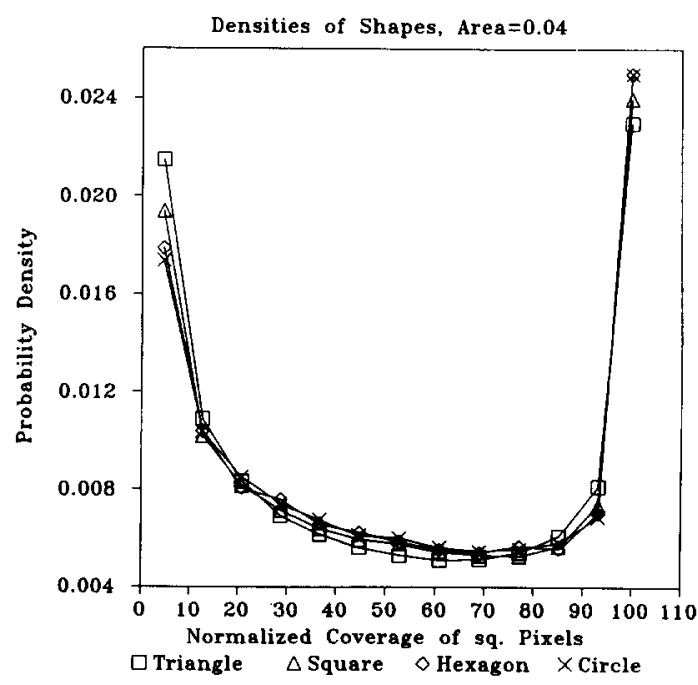

Fig. 8. Densities of different shapes of the same relative 0.04 area. The area of the pixel is 1.0 . The densities are normalized to their maximum abscissa and each of them has 1.0 integral. 
(16-32 intervals) data representation of histograms is enough to get a high measurement accuracy. In practice, usually 15-25 intervals are used in the comparisons.

\subsection{Experiments with circular spots and double circular spots}

In Fig. 3 the histograms of measured circular spots can be seen. The parameter is the ratio of the area of the circle and the area of the sensor-window. Now, the functions are normalized by fitting the derivatives at exposure value $G$ before the right-hand-side maximum, because this peak is a very definite part of the curve (it has a finite probability that the object is inside the pixel, so the density should be a Dirac-delta in the continuous case). In the measurement the estimation accuracy of spot-area was better than 0.03 by using different comparison methods.

In Fig. 4 histograms and densities of double circles are seen. The parameter is the distance between circles relative to the pixel-width. The task is the estimation of this distance. The distance between spots was determined with about 0.10 accuracy.

As it is seen in Figs 3 and 4, the histograms of double and single spots are so different that they can be separated in an easy way.

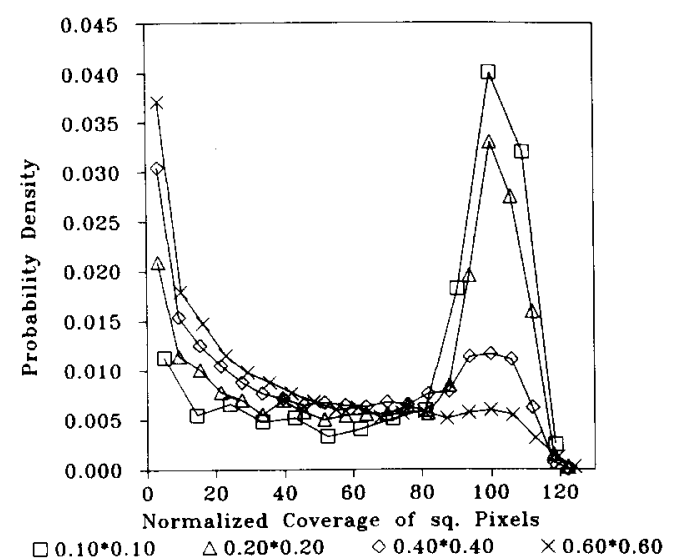

(a)

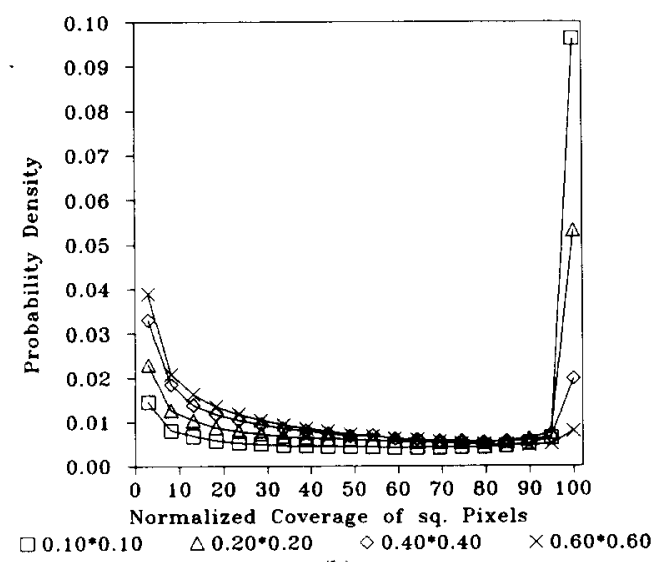

(b)

Fig. 9. (a) Histograms of squares of different relative sidelengths. (b) Densities of the same squares.
This method should be interesting for astronomy (double stars) and laser applications. ${ }^{(11)}$

\subsection{Experiments with squares and rectangles}

In Fig. 9 the histograms and densities of squares can be found. The parameter is the area of the side-length of the square relative to that of the sensor-window. In Fig. 10 the densities of rectangles of different lengthto-width ratios are seen. In Table 1 results of correlation, ML, MDI and L1-distance comparison are shown for squares of different side-lengths.

In Fig. 11 the histograms of double squares are seen. The parameters are the distance between squares and the side-length of one square relative to the width of the pixel-window.

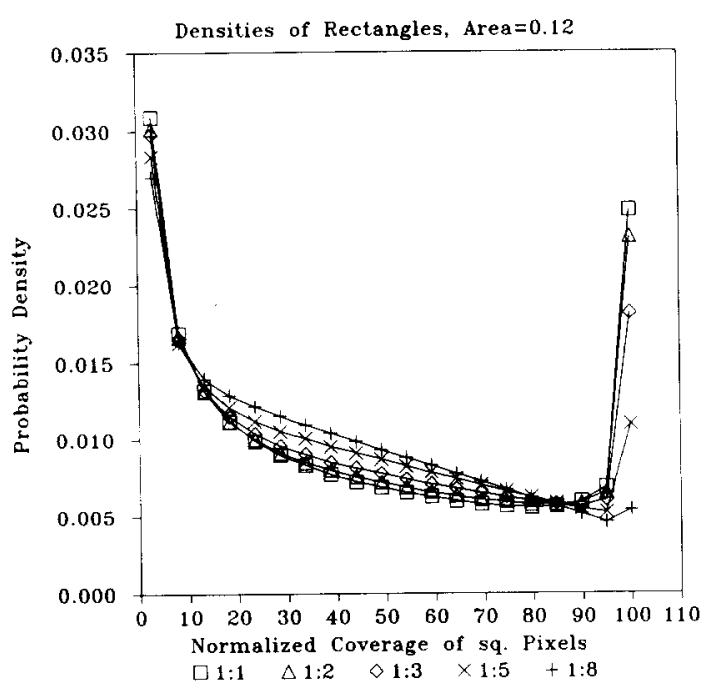

Fig. 10. Densities of rectangles of 0.12 relative area and different length-to-width ratio.

Table 1. Comparison of histograms of squares of different side-lengths (in \%) to hypothetical densities

\begin{tabular}{|c|c|c|c|c|c|c|c|c|}
\hline \multirow{2}{*}{$\begin{array}{l}\text { Hist } \\
\Downarrow\end{array}$} & \multicolumn{4}{|c|}{$M L * 1000$ on hyp. } & \multicolumn{4}{|c|}{ MDI $* 1000$ on hyp. } \\
\hline & 14 & 20 & 30 & 60 & 14 & 20 & 30 & 60 \\
\hline 14 & 4 & 6 & 17 & 83 & 4 & 6 & 16 & 73 \\
\hline 20 & 5 & 2 & 6 & 54 & 5 & 2 & 6 & 49 \\
\hline 30 & 19 & 10 & 4 & 28 & 19 & 10 & 4 & 27 \\
\hline 60 & 87 & 47 & 23 & 2 & 95 & 50 & 25 & 2 \\
\hline
\end{tabular}

\begin{tabular}{|c|c|c|c|c|c|c|c|c|}
\hline \multirow{2}{*}{$\begin{array}{l}\text { Hist } \\
\Downarrow \\
\end{array}$} & \multicolumn{4}{|c|}{$\mathrm{L} 1 * 100$ on hyp. } & \multicolumn{4}{|c|}{ Corr * 1000 on hyp. } \\
\hline & 14 & 20 & 30 & 60 & 14 & 20 & 30 & 60 \\
\hline 14 & 6 & 7 & 12 & 2 & 991 & 985 & 972 & 942 \\
\hline 20 & 7 & 4 & 8 & 22 & 991 & 995 & 993 & 977 \\
\hline 30 & 14 & 10 & 7 & 16 & 976 & 987 & 992 & 987 \\
\hline 60 & 30 & 21 & 14 & 4 & 945 & 982 & 993 & 998 \\
\hline
\end{tabular}




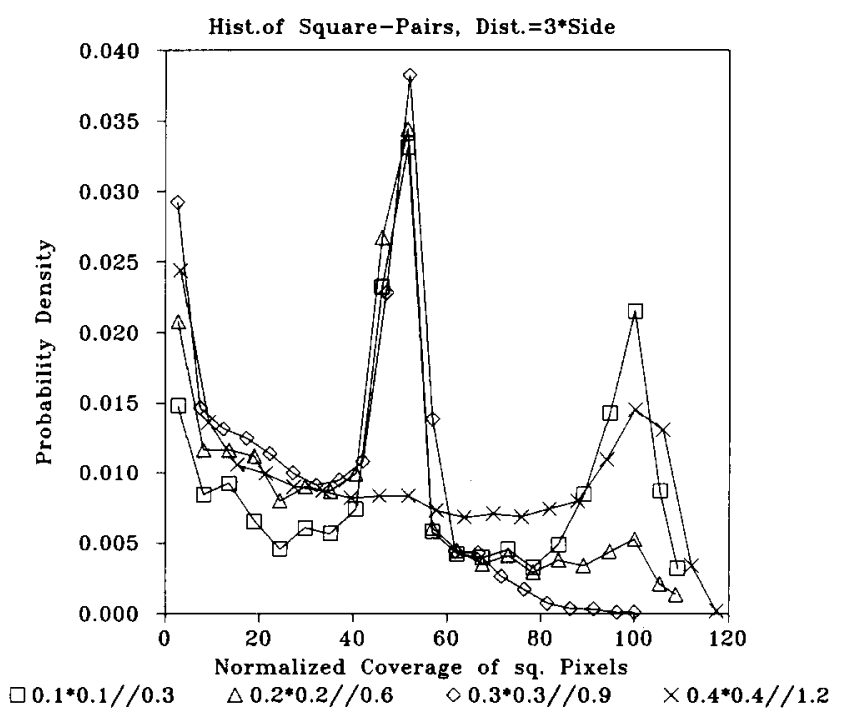

Fig. 11. Histograms of square-pairs of different relative sidelengths and center-distances (distance $=3 *$ side).

3.3. Line drawing analysis: effects of periodically spaced or nonuniformly tilted targets

In another experimental example the measured object is a long curving line with a stable width. The elementary subpixel-size objects are stripe-segments crossing the pixel window. Possible tasks are the recognition of curves of different widths, or the recog-
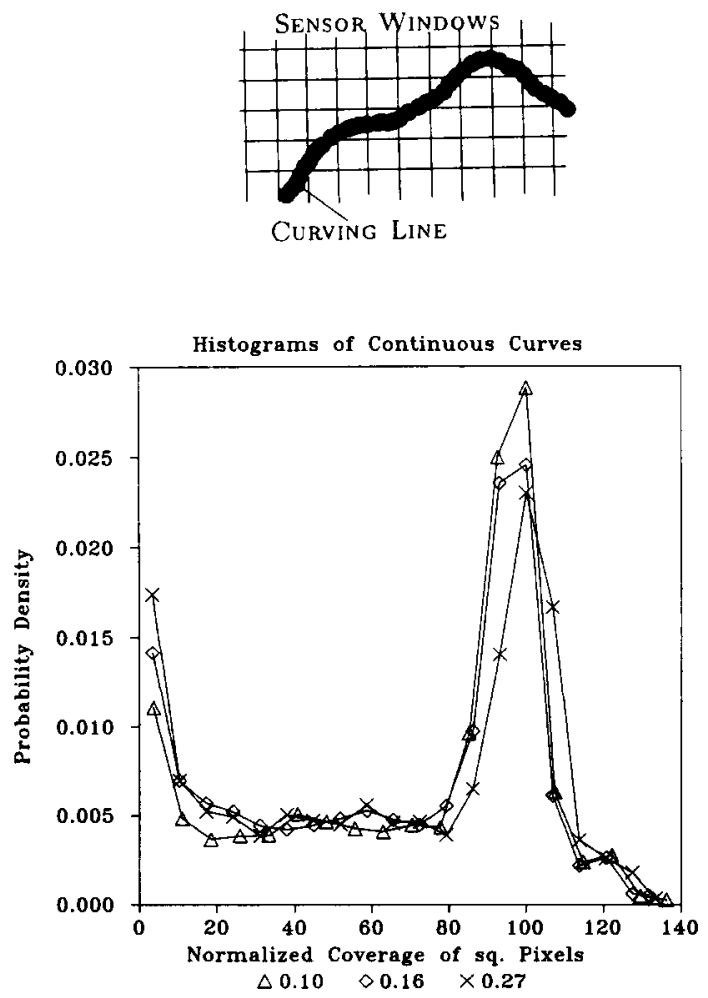

Fig. 12. Histograms of curving continuous lines of different widths. nition of the line type (continuous, dotted, dashed lines).

The calculation of densities is made for the uniform distribution of the deviation angle of the line and distance between line and pixel-center. The track of a stripe going from a pixel to its neighbor is considered a straight line, and the first neighborhoods are considered in the simulations.

The normalized histograms of curving line can be found in Fig. 12. These plots are nearly the same as that of the hypothetical densities. Since there is not a definite right-hand-side peak at the border of the interval, a new normalization is needed for the highest peak going from right to left.

It is found that in the case of (nearly) the same $\omega$ of simulation and measurement, the correlation between the histogram and density is very good. The line-width estimation by the above comparisons gives higher accuracy than $8 \%$ of the window-width. ${ }^{(11,32,34)}$

In Fig. 13 an image of different lines (dotted, sparsely dashed, dense-dashed) is found, in Fig. 5 the measured histograms of the different curve-lines of all angles are plotted. In Fig. 6 the measured histograms of different horizontal lines are shown. It can be seen that the histograms of curving and horizontal lines are different. In this case, the histograms of a curving line can be used as standard histograms (a good estimation of densities) to recognize the horizontal lines. Table 2 compares the measured (horizontal lines) and standardized measured (long curve in all angles) histograms using L1-distance, correlation and the informational divergences of the histograms.

Through the measured lines of Fig. 6 were very short (few hundreds of pixels) and the histograms are "noisy" (partly because of the periodicity of spots), the classification works well even under these circumstances with L1 method in every case. The other three methods 


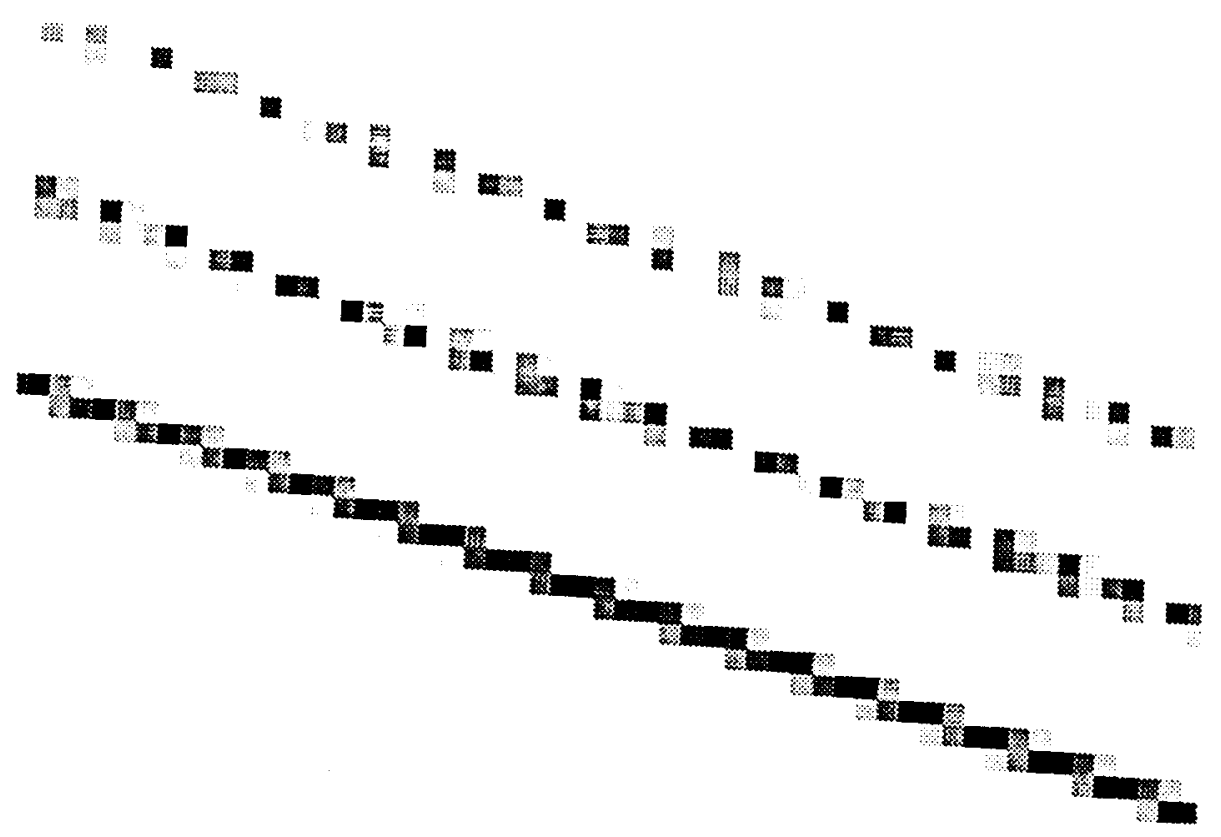

Fig. 13. Image of different lines: dotted, sparsely dashed, dense-dashed.

Table 2. Comparison of histograms of horizontal lines to histograms of curving lines: continuous, dense-dashed, raredashed, dotted. Line-width $=0.20$

\begin{tabular}{|c|c|c|c|c|c|c|c|c|}
\hline \multirow{2}{*}{$\begin{array}{c}\text { Hor } \\
\Downarrow \\
\end{array}$} & \multicolumn{4}{|c|}{$\mathrm{ML} * 100$ on hyp. } & \multicolumn{4}{|c|}{ MDI $* 100$ on hyp. } \\
\hline & $\mathrm{Co}$ & DD & RD & Do & $\mathrm{Co}$ & DD & RD & Do \\
\hline Co & 15 & 26 & 74 & 25 & 17 & 30 & 68 & 27 \\
\hline $\mathrm{DD}$ & 8 & 5 & 5 & 11 & 8 & 5 & 5 & 11 \\
\hline RD & 32 & 29 & 20 & 37 & 49 & 48 & 29 & 49 \\
\hline Do & 61 & 47 & 57 & 33 & 57 & 48 & 75 & 28 \\
\hline
\end{tabular}

\begin{tabular}{lccccccccc}
\hline & \multicolumn{3}{c}{ L1*100 on hyp. } & \multicolumn{3}{c}{ Corr*100 on hyp. } \\
\cline { 2 - 7 } $\begin{array}{l}\text { Hor } \\
\Downarrow\end{array}$ & Co & DD & RD & Do & Co & DD & RD & Do \\
\hline Co & $\mathbf{4 0}$ & 54 & 82 & 50 & $\mathbf{9 2}$ & 81 & 31 & 80 \\
DD & 26 & $\mathbf{2 0}$ & 23 & 32 & 92 & $\mathbf{9 5}$ & 97 & 88 \\
RD & 49 & 48 & $\mathbf{3 8}$ & 55 & 79 & 75 & $\mathbf{8 5}$ & 72 \\
Do & 75 & $\mathbf{6 5}$ & $\mathbf{7 4}$ & $\mathbf{5 1}$ & 91 & $\mathbf{9 8}$ & 94 & $\mathbf{9 9}$ \\
\hline
\end{tabular}

result in some minimal error discriminating sparsely dashed and dense-dashed lines. In this measurement the $\chi^{2}$ test is very poorly satisfied.

\subsection{Recognition of different shapes}

In Fig. 14 and Table 3, densities of different shapes and the results of comparisons are shown. In these experiments small spots formed by laser-printed dots were scanned as objects. These measured granular patterns are compared with the "ideal" computed patterns. The $\chi^{2}$ test gives $75 \%$ for the circles, $88 \%$ for the squares and $96 \%$ for the rectangles. Even in the poor estimation (circles), the recognition works well by using
Table 3. Recognition by comparison of histograms and densities: circle, square, rectangle $1: 2$, triangle. Area $=0.16$

\begin{tabular}{|c|c|c|c|c|c|c|c|c|}
\hline \multirow{2}{*}{$\begin{array}{l}\text { Hist } \\
\Downarrow\end{array}$} & \multicolumn{4}{|c|}{ ML* 1000 on hyp. } & \multicolumn{4}{|c|}{ MDI * 1000 on hyp. } \\
\hline & $\mathrm{Ci}$ & $\mathrm{Sq}$ & $\operatorname{Re}$ & $\operatorname{Tr}$ & $\mathrm{Ci}$ & $\mathrm{Sq}$ & $\operatorname{Re}$ & $\operatorname{Tr}$ \\
\hline & 3.5 & 5.3 & 3. & 12 & 3. & 5.2 & 3. & 12 \\
\hline Sq & 4.8 & 2.9 & 3.3 & 4.6 & 4.7 & 2.8 & 3.2 & 4.5 \\
\hline $\operatorname{Re}$ & 7.0 & 6.5 & 4.1 & 11 & 6.9 & 6.3 & 4.1 & 11 \\
\hline
\end{tabular}

\begin{tabular}{|c|c|c|c|c|c|c|c|c|}
\hline \multirow{2}{*}{$\begin{array}{l}\text { Hist } \\
\Downarrow\end{array}$} & \multicolumn{4}{|c|}{ L $1 * 100$ on hyp. } & \multicolumn{4}{|c|}{ Corr $* 1000$ on hyp. } \\
\hline & $\mathrm{Ci}$ & $\mathrm{Sq}$ & $\operatorname{Re}$ & $\mathrm{Tr}$ & $\mathrm{Ci}$ & $\mathrm{Sq}$ & $\operatorname{Re}$ & $\mathrm{Tr}$ \\
\hline $\mathrm{Ci}$ & 4.6 & 7.6 & 5.6 & 12 & 993 & 993 & 995 & 989 \\
\hline $\mathrm{Sq}$ & 6.7 & 4.5 & 4.7 & 6.8 & 993 & 997 & 996 & 997 \\
\hline $\operatorname{Re}$ & 7.9 & 7.8 & 6.3 & 11 & 988 & 995 & 996 & 994 \\
\hline
\end{tabular}



Fig. 14. Densities of different shapes of area $=0.16$. 
L1 method. These demonstrative results and other experiments successfully demonstrate the usefulness of the theory.

\section{LIMITS OF SUBPIXEL RECOGNITION}

Theoretically, this method can even discriminate shapes that are not very different (e.g. circle and hexagon). There remained a very important question: What is the practical limit of application in the presence of noise and disturbing background?

This statistical subpixel recognition method has its theoretical and practical limits. For example, a practical limit is the necessity that the statistical distribution of the rotation angle of the object examined should be known for density simulation. If the picture is not uniformly illuminated, the pixel gray-levels should be normalized. When the parameters of the scanned patterns are varying as a function of picture position, this function should be considered in the density simulation (e.g. when the optical magnification is changing when moving the optical axis away).

The simulation is not necessary when reliable standard measurements are possible prior to the classification to estimate the densities of different classes. In this way, the simulation of noise-effects can be avoided, but this standardized density estimation ${ }^{(24,25)}$ needs higher measurement accuracy.

Patterns of different classes must not be mixed. However, there are several cases in machine vision field where subpixel patterns of the same class can be separated from the others. In the following, some examples are given:

- In industrial laser applications the parameters of followed laser-beam can be measured ${ }^{(10,1)}$ (beam-shape, diameter).

- In astronomical or military applications separated objects (slowly moving) in an image sequence can be recognized.

- In line-drawing analysis at a low-resolution scanning, a line between crossings is recognized by using the histogram of the separated line-segment area.

- When the noisy background is statistically welldefined (or it is separately measurable, see Section 2.3), the considered densities can be estimated through equation (11).

When different patterns generate very similar histograms at a given accuracy, there is no way to discriminate them. So, the discriminatory power of this method is dependent on the separability of densities of different classes at a given measurement accuracy and imagenoise.

\subsection{Subpixel recognition method and image convolution}

Since the pixels are evaluated as single events, the aliasing effect is not considered. Much rather, convolution theory ${ }^{(35)}$ determines our method. The main difference between our method and the convolution is that in the case of convolution the original shape of the object scanned by the windows can be reconstructed via deconvolution technique, while in the case of the recent method, the statistics of the randomized scanning cannot be used to recover the original shape. So, it can be said when the patterns of blurred (convolved) objects of different classes cannot be separated without deconvolution, this statistical method cannot even be used for subpixel pattern recognition. It means that the difference between the blurred images of different classes after the smoothing effect of transmission (through the optical system and the scanning-windows) should be greater than the quantization error.

\subsection{Limits defined in the frequency domain}

In the spatial frequency domain a similar statement can also be given when the frequency spectrum of the projected, scanned and convolved ${ }^{(13,36)}$ (by the scanning-windows) image is considered. The quantization error may be considered as white-noise. ${ }^{(37)}$ In the sampling process using equal quantization of $r$ number of intervals, the classification between two classes of subpixel-size objects cannot be effective in the case when the difference between the frequency spectra of these scanned objects is less than $1 / r$.

\section{CONCLUSIONS}

As can be seen from the previous experiments, this subpixel recognition method works well for several pattern classes, even in a number of cases when the conventional recognition methods are unsuccessful. Theoretically, the efficiency of recognition is limited by the final resolution of the optical system and the accuracy of density estimation only.

The above method can be implemented in any imageprocessing system, because histograms are basic features to be acquired. However, this method is not an improvement of some conventional method, but it is a new measurement technology for conventionally unsolvable problems (recognition in the subpixel range). With this statistical evaluation the resolution accuracy of conventional image processing systems can be increased by a factor of about $10-100$ for a limited number of object-classes, depending on noise and measurement accuracy.

The possible application area of this method is mostly the cases where the background can be eliminated at high signal-to-noise ratio: astronomical measurements, industrial laser applications, robot tactile sensors, images captured by high quality document scanners.

This method is intended to be used in line-drawing analysis systems. A special application area could be the track analysis of cloud tracks in cloud-chamber experiments of high energy nuclear particles. This method may be used in many astronomical and industrial applications with success.

Texture analysing systems can also use this method, but it leads to another field which has been partly 
covered by existing theories. ${ }^{(38)}$ However, some undersampled textures of sparse separated patterns can be analysed by this new method.

The statistical type of recognition should also be considered in natural systems ${ }^{(39)}$ and artificial neural networks. The above results imply that in the environment of "firing" neurons of a natural scanning system, the low-resolution recognition should be rather statistical than some fine convolution method.

Acknowledgements - Special thanks are due to Professor L. Györfi for his helpful criticism. The assistance given by the Computer and Automation Institute, Hungarian Academy of Science and by Professor T. Roska is very much appreciated.

\section{REFERENCES}

1. S. E. Reichenbach, S. K. Park and R. Narayanswamy Characterizing digital image acquisition devices, Opt. Engng. 30, 170-177 (1991).

2. W. L. G. Warmerdam and V. R. Algazi, Describing 1D intensity transitions with Gaussian derivatives at the resolution matching the transition widths, IEEE Trans. Pattern Anal. Mach. Intell. PAMI-11, 973-977 (1989).

3. E. p. Lyvers, O. R. Mitchell, M. L. Akey and A. P. Reeves, Subpixel measurement using a moment based edge operator, IEEE Trans. Pattern Anal. Mach. Intell. PAMI-11, 1293-1309 (1989).

4. I. J. Cox, J. B. Kruskal and D. A. Wallach, Predicting and estimating the accuracy of a subpixel registration algorithm, IEEE Trans. Pattern Anal. Mach. Intell. PAMI-12, 721-734 (1990).

5. J. Koplowitz and P. Sundar Raj, A robust filtering algorithm for subpixel reconstruction of chain coded line drawings, IEEE Trans. Pattern Anal. Mach. Intell. PAMI-9, 451-457 (1987).

6. B. F. Alexander and K. C. Ng, Elimination of systematic error in subpixel accuracy centroid estimation, Opt. Engng 30, 1320-1331 (1991).

7. A. Huertas and G. Medioni, Detection of intensity changes with subpixel accuracy using Laplacian-Gaussian masks, IEEE Trans. Pattern Anal. Mach. Intell. PAMI-8, 651-664, (1986).

8. H. K. Aghajan, C. D. Schaper and T. Kailath, Machine vision techniques for subpixel estimation of critical dimensions, Opt. Engng 32, 828-839 (1993).

9. T. Szirányi, P. Barczikay and T. Kovács, Measurement theory of laser-beam diameter of about $1 \mu \mathrm{m}$ by moving CCD-sensor, Int. J. Optoelectr. 3, 187-194 (1988).

10. T. Szirányi, L. Illés and T. Kovács, Procedure and arrangement to determine a beam-diameter, Invention, Serial No.: 201403, Budapest (1990).

11. T. Szirányi, Statistical subpixel measurement technology of light-beam width and profiles, Optics Lasers Engng 16 . $1-15$ (1992).

12. W. K. Pratt, Digital Image Processing. Wiley, New York (1991).

13. T. Szirányi and Á. Böröczky, Overall picture degradation error for scanned images and the efficiency of character recognition, Opt. Engng 30, 1878-1885 (1991).

14. T. Szirányi, Statistical pattern recognition of low resolution pictures, Pattern Recognition Lett. 8, 221-228 (November, 1988).

15. R. Bajcsy, G. Buttazzo and P. Dario, Finger based explorations, in Proc. SPIE, 726, pp. 338-345 (1986).

16. C. O. Fairchild, Charge Coupled Device (CCD) Catalog, (1984).
17. Texas Instruments, Optoelectronics and Image-sensor Data Book, Dallas, Texas (1987).

18. EG \& G Reticon, Image Sensing Products 1992/1993, Sunnyvale, CA (1992).

19. H. Shibata, I. Inoue, R. Miyagawa, H. Yamashita, N. Nohmi, A. Furukawa, Y. Iida, T. Yamaguchi, Y. Endo, Y. Matsunaga and S. Manabe, 2M-pixel vertically-integrated HDTV image sensor, Abstract Int. Solid-State Circuits Conf. (39th ISSCC), February, 1992, San Francisco (IEEE) (1992)

20. R. A. Hummel, Histogram modification techniques, Comput. Graphics Image Process. 4, 209-224 (1975).

21. A. K. Jain, Fundamentals of Digital Image Processing. Prentice-Hall Int. Inc., Englewood Cliffs (1989)

22. H.S. Wong, Effect of knife-edge skew on modulation transfer function measurements of charge coupled device imagers employing a scanning knife edge, Opt. Engng 30, 1394-1398 (1991).

23. I. Csiszár, Information-type measures of difference of probability distributions and indirect observations, Studia Scientiarum Mathematicarum Hungarica 2, 299-318(1967).

24. L. Devroye, A Course in Density Estimation. Birkhauser, Boston (1987).

25. L. Györfi, W. Hardle, P. Sarda and P. Vieu, Nonparametric Curve Estimation from Time Series (in Lecture Notes in Statistics, No. 60.). Springer, New York (1989).

26. A. R. Barron, L. Györfi and E. Meulen, Distribution estimation in total variation and in informational divergence IEEE Trans. Information Theory. IT-38, 1437-1454(1992).

27. L. Györfi, I. Vajda and E. C. Meulen, Family of point estimates yielded by L1-consistent density estimate, in Proc. Second Int. Conf. on Statistical Data Analysis Based on the L1-Norm and Related Methods (Neuchatel, Switzerland), pp. 415-430 Elsevier Sci. Publ., London 17-20 August (1992).

28. S. Kullback and R. A. Leibler, On information and sufficiency, Ann. Math. Statist. 22, 79-86 (1951).

29. I. Csiszár, About the MDI, in Multivariable Statistical Analysis (in Hungarian), edited by F. T. Móri and J. G. Székely, pp. 209-232. Müszaki Könyvkiadó, Budapest (1986).

30. G. A. Korn and T. T. Korn, Mathematical Handbook for Scientists and Engineers. McGraw-Hill, New York (1975)

31. I. Csiszár and J. Körner, Information Theory. Academic Press, Budapest (1981).

32. T. Szirányi and J. Csicsvári, Subpixel technology for pattern recognition and measurement of known shapes, in Proc. 3rd Workshop on Hungarian Picture Processing, MTESz, Budapest, 32-39 (1991)

33. L. Devroye, Consistent deconvolution in density estimation, Can. J. Statistics-Revue 17, 235-239 (1989).

34. T. Szirányi, New measurement and recognition methods in picture processing, (in Hungarian), $\mathrm{Ph} . \mathrm{D}$. dissertation, Hungarian Academy of Sciences, Budapest, pp. 5-41 (1991).

35. G. W. Stroke and R. G. Zech, A posteriori image-correcting 'deconvolution' by holographic Fourier-transform division, Phys. Lett. 25A, 89-90 (1967).

36. W. J. Smith, Modern Optical Engineering. McGraw-Hill, New York (1966).

37. I. Kollár, Tools and methods of signal analysis, in Technology of Electrical Measurements, edited by L. Schnell. John Wiley \& Sons Ltd, London (1992).

38. R. M. Haralick and K. Shanmugam, Textural features for image classification, IEEE Trans. Systems Man Cybernet. SMC-3, 610-621 (1973).

39. P. Baldi and W. Heiligenberg, How sensory maps could enhance resolution through ordered arrangements of broadly tuned receivers, Biol. Cybernet. 59, 313-318(1988). 
About the Autbor-TAmás SzIRÁNYı received the Diploma and the Dr. Techn. degrees in electronics technology from the Technical University in Budapest in 1980 and 1983, respectively. He received the Ph.D. degree in electronics and computer engineering in 1991 from the Hungarian Academy of Sciences.

Until 1991, he was a Research Fellow and Project Manager of Optoelectronics Development at the VIDEOTON Development Institute, Budapest. Then his work involved designing of optical data storage, laser printer and document scanning devices, and also applications of laser systems. He led a research department and organized several projects. At the same time, he was a lecturer in electronics and computer technology at the Technical University in Budapest for several years.

$\mathrm{He}$ is currently a Senior Research Fellow at the Computer and Automation Research Institute of Hungarian Academy of Sciences, and he is an Advisory Professor of image analysis at the Information Technology Department of the Veszprem University.

His research activities include pattern and texture analysis with cellular neural networks, information theory of picture processing, character recognition, subpixel measurement techniques and hardware research. He designs systems including optoelectronic devices, analog and digital hardware and DSPs.

He has published more than 30 scientific papers concerning image analysis, cellular neural networks and optoelectronic systems. He is a member of (IEEE, SPIE and d.v. Neumann Computer Society. 